EPJ Web of Conferences 47, 01007 (2013)

DOI: $10.1051 /$ epjconf/20134701007

(C) Owned by the authors, published by EDP Sciences, 2013

\title{
Classification of variable stars in the WFCAM Transit Survey
}

\author{
Hristo Stoev ${ }^{1, a}$, David Barrado ${ }^{2,1}$, Luis M. Sarro ${ }^{3}$ and Andrés Moya ${ }^{1}$ \\ ${ }^{1}$ Departamento de Astrofísica, Centro de Astrobiología (CSIC-INTA), PO Box 78, \\ 28691 Villanueva de la Cañada, Spain \\ ${ }^{2}$ Calar Alto Observatory, Centro Astronómico Hispano-Alemán, cl Jesús Durbán Remón, 2-2, \\ 04004 Almería, Spain \\ ${ }^{3}$ Departamento de Inteligencia Artificial, Universidad Nacional de Educación a Distancia, \\ cl Juan del Rosal, 16, 28040 Madrid, Spain
}

\begin{abstract}
The WFCAM Transit Survey is a photometric survey in the near-infrared and aims at finding Earth-like planets transiting M-dwarf stars. As a by-product of the survey, a variety of variable stars has been detected. We report the discovery and classification of 192 periodic variable stars in the WFCAM Transit Survey. 185 of those objects are previously unknown variable sources. The derived parameters of their light curves will be helpful for the creation of a robust sample of light curves (and their parameters thereof) of classified variable stars in the near-infrared for the automatic classification of light curves of stellar objects in the J-band.
\end{abstract}

\section{INTRODUCTION}

The studies of variable stars have been one of the main fields of research in astronomy. In recent years, time-resolved photometric surveys have marked a memorable boom in time-domain astronomy. Projects such as OGLE, ASAS and Pan-STARRS are constantly monitoring the night sky from Earth and as a byproduct, catalogs of variable stars are created. The space missions CoRoT and Kepler, dedicated to the search of transiting extrasolar planets and asteroseismology, have also produced detailed censuses of the variable star population in their fields. With the ongoing Vista Variables in the Vía Lactea (VVV) ESO Public Survey with an expected detection of $\sim 10^{6}$ variable point sources and the soon-to-be-launched Gaia spacecraft, which will monitor more than $10^{9}$ objects over the whole sky allowing the discovery of millions of variable stars [1], all those new detected variable sources will need to be properly classified.

\section{THE WTS SURVEY}

The WFCAM Transit Survey is an ongoing photometric survey campaign operating on the 3.8-m United Kingdom Infrared Telescope (UKIRT) since August 2007. The survey is monitoring 4 target fields, each of which covering 1.5 square degrees of sky, with the Wide-Field Camera imaging array in the infrared wavelengths (J-band, $1.25 \mu \mathrm{m})$ [2].

Each of the fields is observed by conducting eight pointings of the WFCAM pawprint. Then they are tiled together in order to provide a uniform coverage across the field. The cadence of the resulting light curves is 4 data points per hour. Depending on the weather conditions, the observations are usually carried out at the beginning of the night in $>1$ " seeing when the atmosphere is still cooling and settling.

\footnotetext{
ae-mail: hstoev@cab.inta-csic.es
}

This is an Open Access article distributed under the terms of the Creative Commons Attribution License 2.0, which permits unrestricted use, distribution, and reproduction in any medium, provided the original work is properly cited. 


\section{EPJ Web of Conferences}

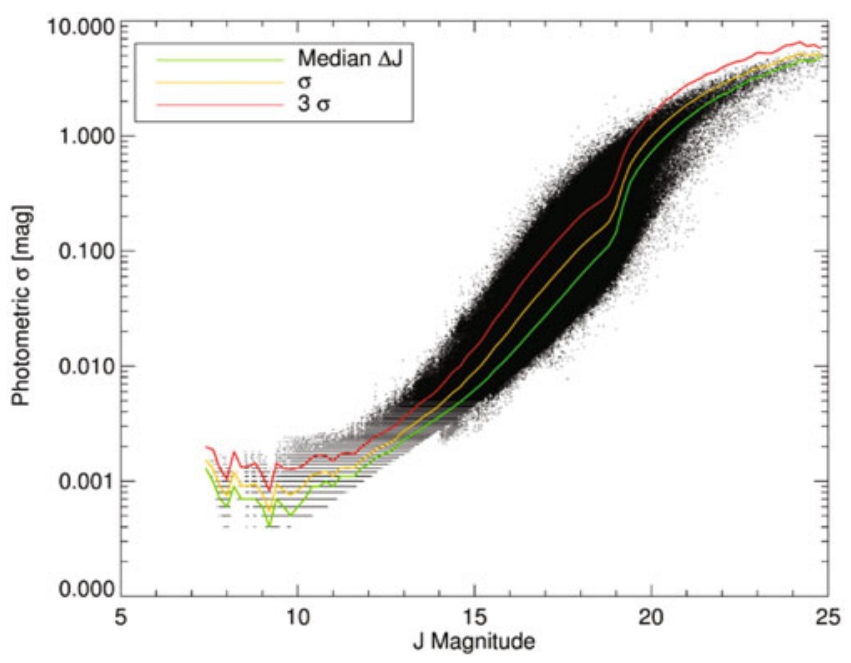

Figure 1. A graphical representation of the distribution of error as a function of the magnitude. The yellow line represents the median error $(\Delta \mathrm{J})$ for each 0.05-magnitude bin; the red line plots the standard deviation $\sigma$ as a function of magnitude and the green line is the $3 \sigma$ level of variation of the error with respect to the median error per bin.

Initial reduction of the raw images is performed using the Cambridge Astronomical Survey Unit (CASU) pipeline, based on the INT wide-field survey pipeline [3]. A so-called "master frame" is created by stacking the best 20 images based on their point-spread function (PSF). Source detection is performed on this image and then by means of aperture photometry, the brightness of each object detected on the master frame is measured on the rest of the frames and a catalogue is generated. At the end of the cycle, the data is written on quadruple-extension FITS binary table, i.e. one FITS container per detector.

The variable stars that are reported in this publication are all found in one of the WTS fields, approximately centered on the following coordinates: $\alpha=19^{h} 35^{m}, \delta=+36^{\circ} 30^{\prime}$, hereafter referred to as the $19 \mathrm{hr}$ field.

\section{DATA SET}

\subsection{Data Characterisation and Reduction of the WTS Light Curves}

The $19 \mathrm{hr}$ field is the field with most extensive coverage and constitutes of 1145 epochs as of June 16, 2011. The majority of the detected objects in the field have a J-magnitude between 15 and 20. At the dimmer end the sensitivity rapidly decreases. Saturation of the detectors occurs at $J \sim 11-12$ mag but this value varies with sky conditions. The photometric errors are automatically estimated by the CASU pipeline on a per-datapoint basis taking into account a model consisting of the Poisson noise in the object counts, the Poisson noise in the sky, the RMS of the sky background fit and a constant component which accounts for systematic noise [4].

Figure 1 presents the variation of the photometric error as a function of the magnitude on a perdatapoint basis.

In the figure, each point represents a measurement of the light curves of all objects which have been classified by the reduction algorithm as a non-blended stellar or probably stellar object. Following [5], the WFCAM J-band gets saturated at $J=11 \mathrm{mag}$. This is also observed on Fig. 1: the distribution levels off at $J=11.5-12 \mathrm{mag}$. The brightest stars have a precision of $\sim 3 \mathrm{mmag}$ and the errors are well-behaved until $J=15 \mathrm{mag}$ where the median error is still under $1 \%$. It should also be noted that 


\section{Hot Planets and Cool Stars}

a $1 \%$ precision is achieved for data points as $\operatorname{dim}$ as $J=17 \mathrm{mag}$. However, on the fainter end, the precision quickly decreases and at $J=19$ mag it reaches as much as 1 mag. Thus any datapoints above this threshold would not provide any meaningful information to the individual light curves. It is also observed that there are many data points well above the $3 \sigma$ level which are most probably obtained during observations under less-than-optimal conditions.

Based on this, and in order to reduce the quantity of false positive detections, a number of steps are undertaken to rest assured that we have as clean a sample as possible. All datapoints outside the $3 \sigma$ interval are removed and after a check that the light curve consists of at least 20 valid magnitude measurements, whose median is smaller than $J=19$ mag, any individual measurements outside the $3 \sigma$ interval on a light curve by light curve basis are rejected.

\subsection{Frequency analysis}

The light curves which successfully pass the filtering are then fed into a procedure which performs a frequency analysis. The selected computer programs are bossirr and freq. They are performing leastsquare fitting techniques following the methodology described in [6] and [7]. The program bossirr fits a number of simultaneous sinusoidal variations in the magnitude domain where the test frequency $\omega$ is iteratively selected from a list of pre-defined possible frequencies. The aim of the program is to minimize

$$
\chi^{2}=\sum_{i=1}^{N}\left[m_{i}-f\left(t_{i}\right)\right]^{2}=\sum_{i=1}^{N}\left[m_{i}-a_{0}-a \sin \left(\omega t_{i}+\phi\right)\right]^{2},
$$

where $m_{i}$ is the brightness measurement at moment $t_{i}, a_{0}$ is a parameter and can be related to the mean value of the inspected signal and $\chi^{2}$ corresponds to the best-fit solution of the model parameters. This method is used to detect up to 2 significant frequencies (the significance level is hard-coded to $99.9 \%$ ) and does not involve subtraction over an average or pre-whitening and is more appropriate given that signals at low frequencies might be expected [8].

The second program, freq, determines up to four harmonic modes of the already-detected frequencies $\omega_{i}$ by bossirr. Using least-square fitting, harmonic functions of the form

$$
f(t)=a \sin (n \pi \omega t+\phi)
$$

are fit to the data to obtain the values of $a_{i j}$ and $\phi_{i j}$ for each $\omega_{i}$. The indices $j$ correspond to the $j$-th amplitude and phase of the harmonics of the frequency $\omega_{i}$. As an optimal final result, for each star there will be two detected significant frequencies with four harmonic modes each, having the amplitude and phase difference for each.

\section{RESULTS}

After a careful visual examination of the phase-folded light curves, 192 new periodic variable stars have been detected in the $19 \mathrm{hr}$ field of which 185 are previously unknown. Based on the morphology of the light curves, the stars were classified into various variability classes, summarised in Table 1 . Figure 2 presents a histogram of the number of detected variables classified as belonging to each of the types.

The majority of the periodic variable stars detected are eclipsing binaries of various types: all of them make up to more than $75 \%$ of the variable stars detected. The following biggest group is the long-period variables. As it can be observed on the histogram presented on the left panel of Fig. 3, the majority of detected variable stars have periods ranging from less than a day to about four days. However, the group of long-period variable stars with periods over 100 days can also clearly be identified on the right-hand end of the histogram. The values of the median magnitudes of the detected variable stars range from $J=12.3$ mag to $J=18.9$ mag. The right panel of Fig. 3 shows a histogram of the median magnitudes showing a peak at around $J=15.7 \mathrm{mag}$. 


\section{EPJ Web of Conferences}

Table 1. Variability classes used in the Catalogue of periodic variable stellar sources in the $19 \mathrm{hr}$ field of the Wide Field Transit Survey.

\begin{tabular}{ll}
\hline Abbreviation & Variability class \\
\hline \hline EA & Algol-type eclipsing system \\
EB & $\beta$ Lyrae-type eclipsing system \\
EW & W Ursae Majoris eclipsing variable \\
LPV & Long-period pulsating variable star \\
M & Mira-type ( $o$ Ceti) variable \\
RRAB & RR Lyrae with asymmetric light curve \\
X & A class representing other types of variability \\
\hline
\end{tabular}

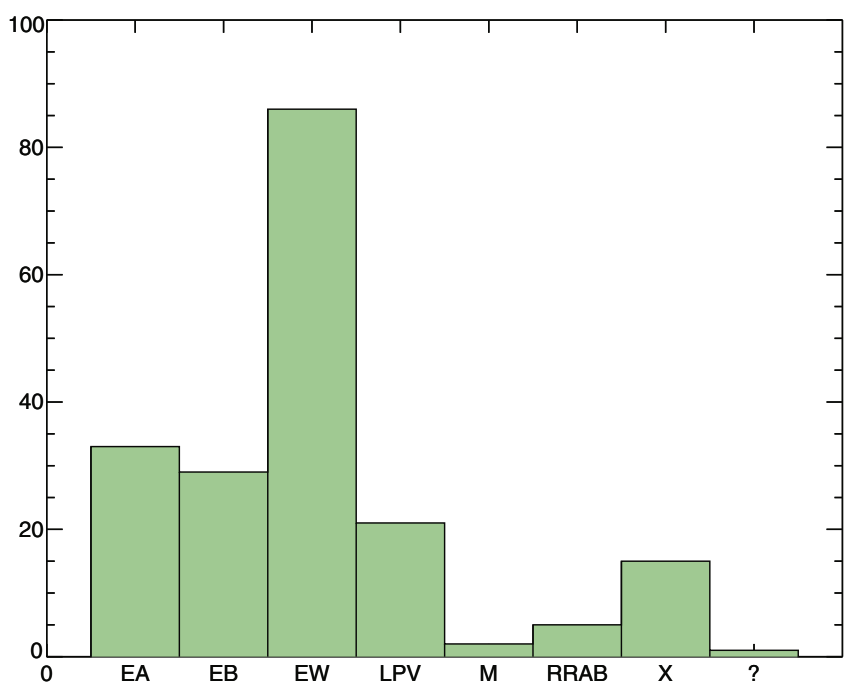

Figure 2. A histogram of the number of stars classified as belonging to the different variability classes. See text for details on type $X$.
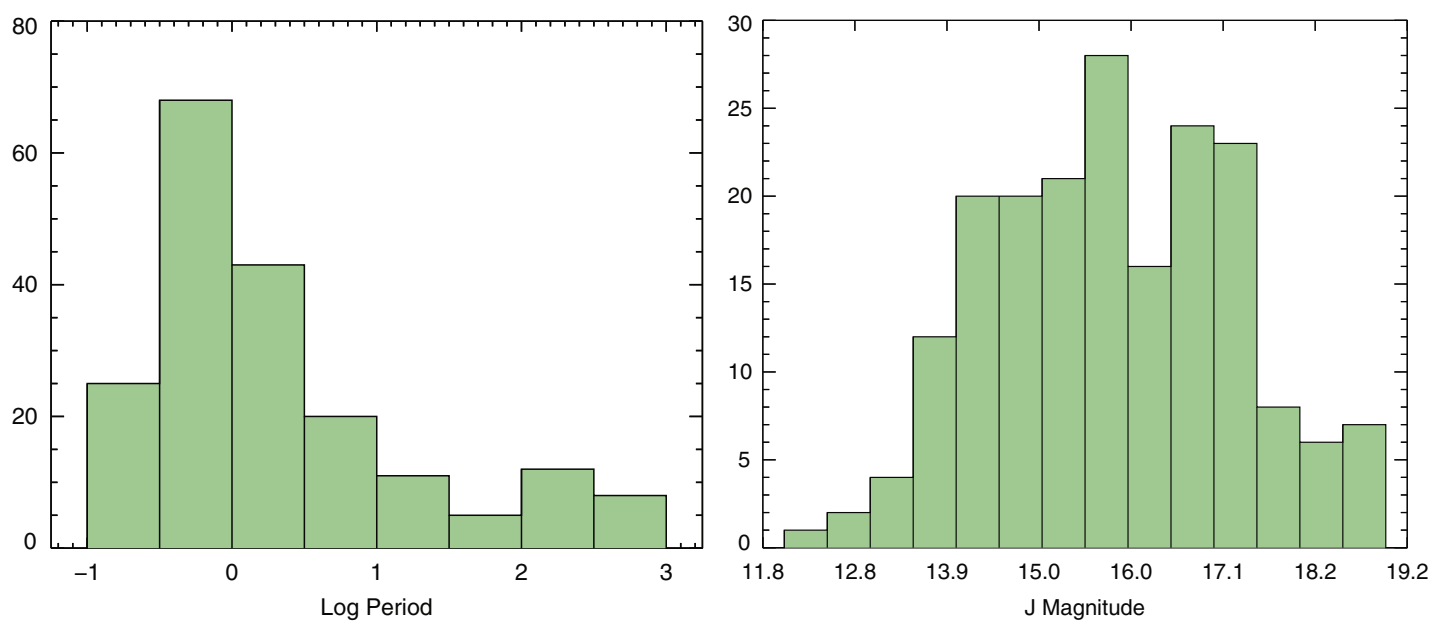

Figure 3. Left panel: histogram of the logarithm of the periods of the detected variable stars; Right panel: histogram of the mean magnitudes of the detected variable stars spanning from $12.3 \mathrm{mag}$ to $18.9 \mathrm{mag}$ with a peak at around $15.7 \mathrm{mag}$ and another less pronounces at $17 \mathrm{mag}$. 


\section{Hot Planets and Cool Stars}
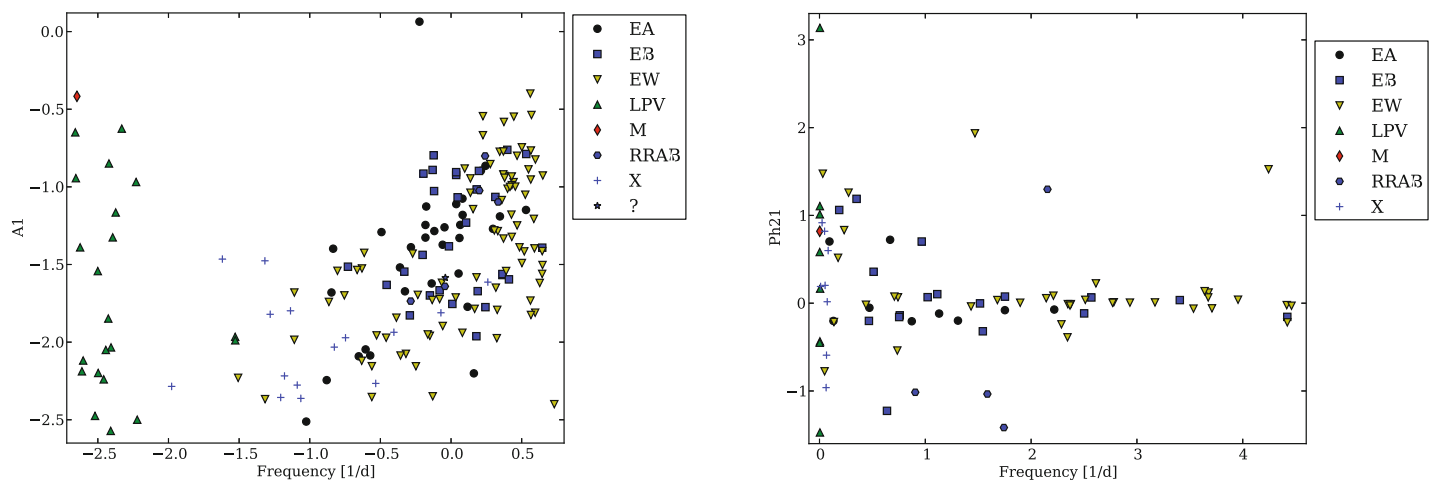

Figure 4. Left panel: logarithm of the amplitude of the first harmonic versus the logarithm of the frequency; Right panel: two-dimensional projection of the frequency and the phase difference parameter space. The $\mathrm{x}$-axis represents the frequency in cycles per day and the y-axis - the phase difference between the first two harmonics of the frequency.

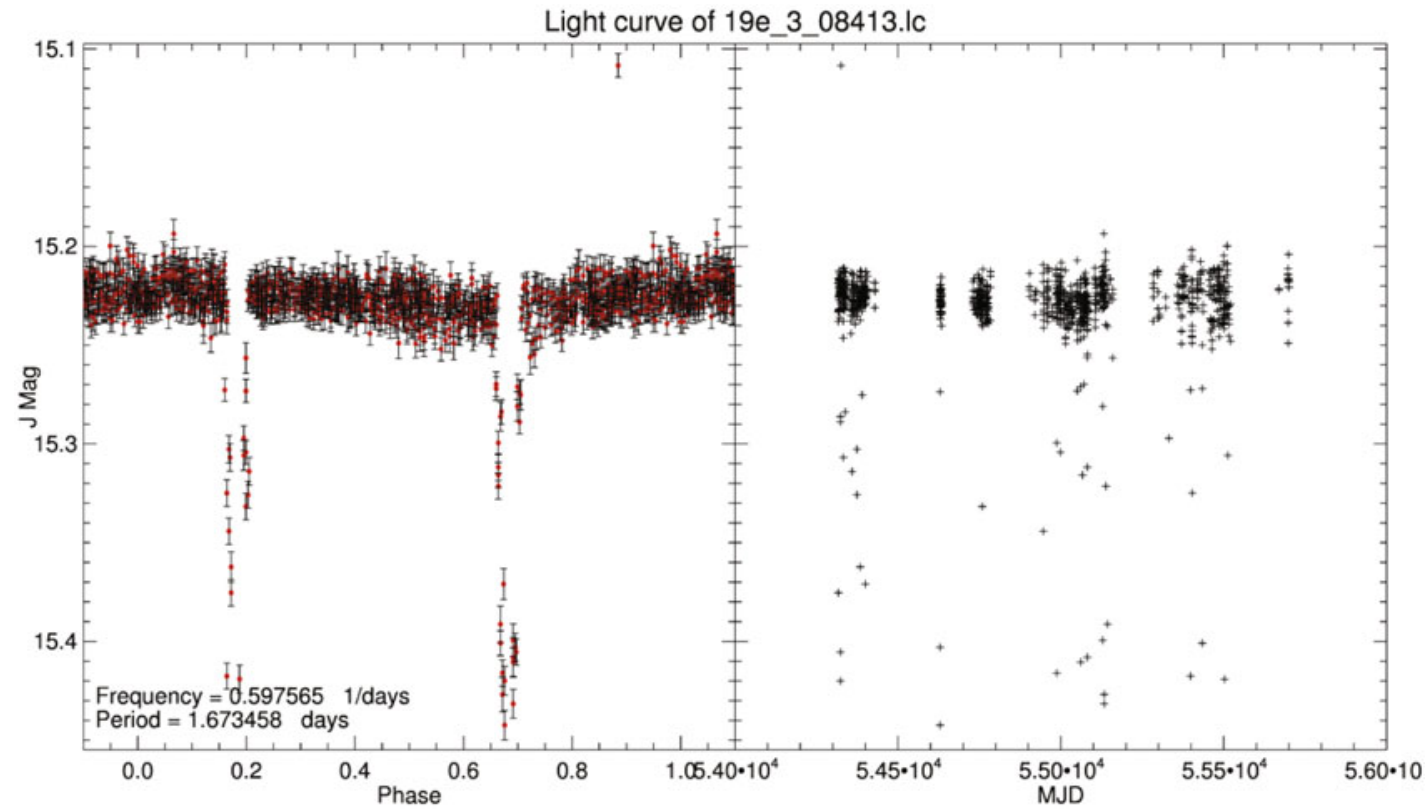

Figure 5. A detached contact binary found in the database with clearly defined primary and secondary minima.

Previous studies $[9,10]$ have demonstrated that various parameters resulting from the analysis of the light curves can be used to successfully classify the objects in one class or another. For example, optical data from Hipparcos, OGLE and CoRoT have been used for the determination of robust sets of parameters derived from the light curves of known member stars of various variability classes. However, such data sample in the near-infrared is unavailable at the moment and it is not straightforward to assume that the parameters will behave in a similar fashion in other wavelength bands. Thus, for a database of near-IR time series observations to be automatically classified in the future, the need arises for a clear 


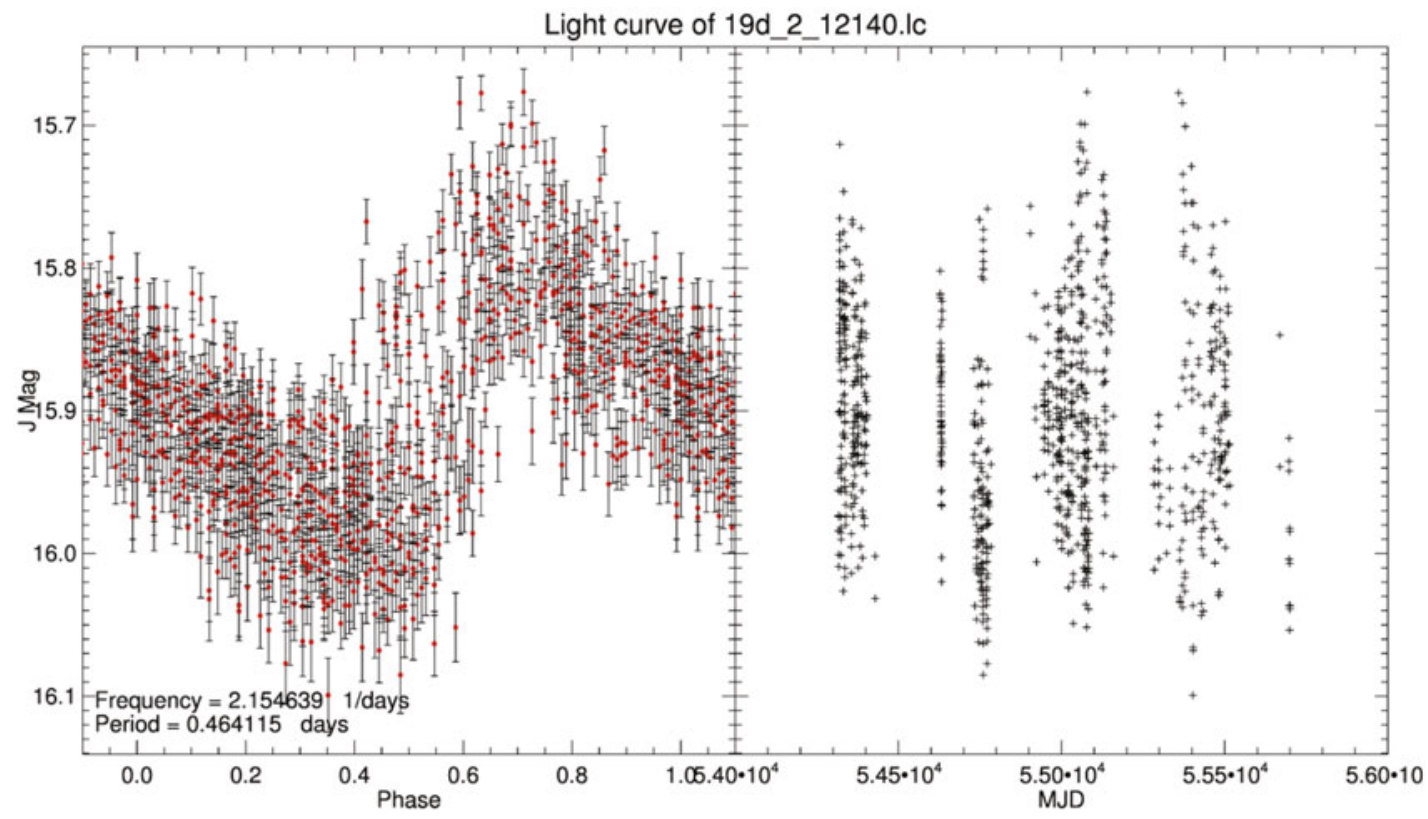

Figure 6. One of the asymmetric periodic variables of type RR Lyr discovered in the database.

definition of the set of parameters of those specific classes so that the new objects in the database are assigned to one of them with a certain probability. For this, let us explore the behaviour of some of the parameters presented, in particular, the frequency, the amplitude, the phase differences between the first two harmonics of the frequency $\left(\phi_{21}=\arctan \left(\sin \left(\arctan \phi_{2}-\arctan \phi_{1}\right), \cos \left(\arctan \phi_{2}-\arctan \phi_{1}\right)\right)+\right.$ $\pi / 2)$ and the ratio between the amplitudes of the first two harmonics $\left(\mathrm{R}_{21}=A_{2} / A_{1}\right)$, in various parameter spaces.

On the left panel of Fig. 4 the logarithm of the amplitude of the first harmonic is plotted against the logarithm of the frequency. Based on this, one can clearly separate the long-period variables from the remaining of the classes, but it is difficult to determine any clustering of other classes of variable objects. On the right panel of Fig. 4 the frequency is plotted versus the phase difference between the first two harmonics of the frequency $\left(\phi_{21}\right)$. What we can observe is that whereas for low frequencies it is not trivial for any clear relation to be detected, the high-frequency domain is dominated by eclipsing binaries of all three classes with $\phi_{21} \approx 0$. This is in accordance with the results from the analysis of the Hipparcos archive presented in [10].

Due to ambiguity in the determination of class allocation based merely on the morphology of the light curves, the variability class $X$ contains objects which may belong to various types, among which are cepheids, ellipsoidal variables and spotted stars showing rotational variability. A study of the spectral energy distributions (SED) of the objects has been initiated and the primary results lead to the conclusion that even though some candidates may present features which could be interpreted as belonging to other variability classes, such as $\delta \mathrm{Scu}, \gamma$ Dor, or SPB, they can be rejected due to the results of the SED fitting performed.

At the end, two light curves, one of a detached eclipsing binary and another - of a RR Lyrae, are presented on Figs. 5 and 6. On Fig. 2 there is one object classified as ?. We detected this variable star with a period of 1.09 days that was impossible to place in any of the classes mentioned above. The light curve of this object is shown on Fig. 7. 


\section{Hot Planets and Cool Stars}

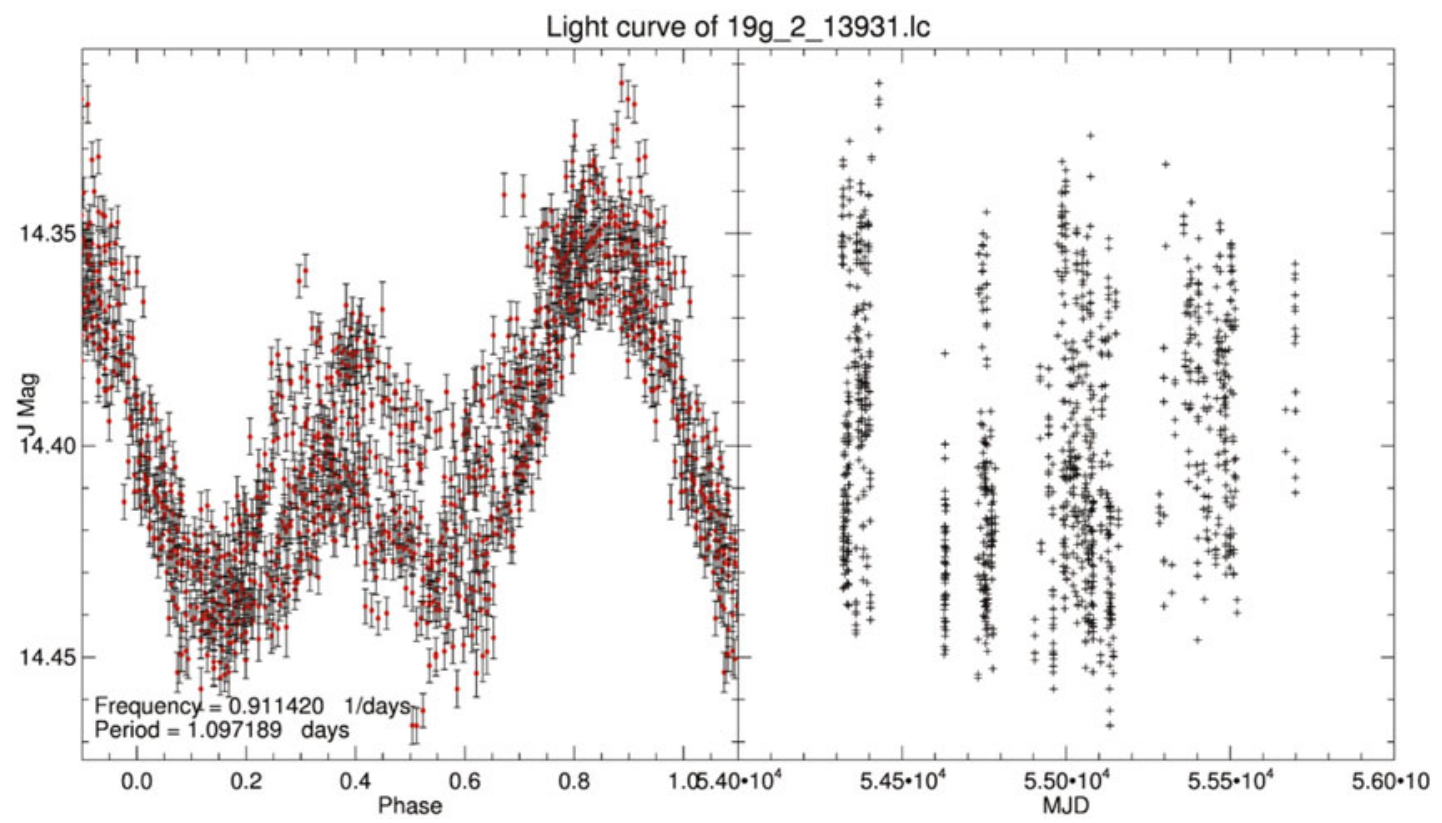

Figure 7. A variable star with a period of 1.09 days that we were unable to classify.

\section{CONCLUSIONS}

We have presented the detection and morphological classification of 185 new variable stars in the WFCAM Transit Survey based on near-IR photometry. Moreover, we also confirm the the classification and periods of another seven stars that are already in the VSX catalogue [11].

The results can be used as a base for creating a training set for the supervised classification of the rest of the WTS fields so that at the end, the periodic variable stars detected in the survey may present a full set for variable star classification in the near-IR.

HS is supported by RoPACS, a Marie Curie ITN funded by the Seventh Framework Programme of the European Commission. This research has been funded by Spanish grants AYA2012-38897-C02-01, and PRICIT-S2009/ ESP-1496.

\section{References}

[1] L. Eyer, J. Cuypers, Predictions on the Number of Variable Stars for the GAIA Space Mission and for Surveys such as the Ground-Based International Liquid Mirror Telescope, in IAU Colloq. 176: The Impact of Large-Scale Surveys on Pulsating Star Research, edited by L. Szabados, D. Kurtz (2000), Vol. 203 of Astronomical Society of the Pacific Conference Series, pp. 71-72

[2] J. Birkby, B. Nefs, S. Hodgkin, G. Kovács, B. Sipőcz, D. Pinfield, I. Snellen, D. Mislis, F. Murgas, N. Lodieu et al., MNRAS 426, 1507 (2012)

[3] M. Irwin, J. Lewis, New Astronomy 45, 105 (2001)

[4] G. Kovacs, S. Hodgkin, B. Sipocz, D. Pinfield, in prep. (2013) 
[5] S.T. Hodgkin, M.J. Irwin, P.C. Hewett, S.J. Warren, MNRAS 394, 675 (2009)

[6] P. Vaníček, ApSS 12, 10 (1971)

[7] J.D. Scargle, ApJ 263, 835 (1982)

[8] F.M. Zerbi, R. Garrido, E. Rodriguez, K. Krisciunas, R.A. Crowe, M. Roberts, E.F. Guinan, G.P. McCook, J. Sperauskas, R.F. Griffin et al., MNRAS 290, 401 (1997)

[9] J. Debosscher, L.M. Sarro, C. Aerts, J. Cuypers, B. Vandenbussche, R. Garrido, E. Solano, A\&A 475, 1159 (2007)

[10] L.M. Sarro, J. Debosscher, C. Aerts, M. López, A\&A 506, 535 (2009)

[11] C.L. Watson, Society for Astronomical Sciences Annual Symposium 25, 47 (2006) 